\title{
PAROXYSMAL NOCTURNAL HEMOGLOBINURIA
}

\author{
Paudyal B P*, Zimmerman M ${ }^{*}$, Karki A*, Neupane $H^{*}$, Kayastha ${ }^{\star}$ \\ * Patan Hospital, Lalitpur, Nepal
}

\begin{abstract}
Paroxysmal nocturnal hemoglobinuria (PNH) is a rare hemolytic disorder of acquired origin and is clinically manifested by chronic hemolysis, thromboses in various sites, and bone marrow failure. The disease is so rare that the delay in the diagnosis is not uncommon and this bears a tremendous impact on patient management. We present this case to draw attention to this uncommon cause of hemolytic anemia, which should be considered in any patient, of any age, who has signs of chronic hemolysis.
\end{abstract}

\section{Key Words: Paroxysmal Nocturnal Hemoglobinuria, Hemolytic anemia.}

\section{INTRODUCTION}

Paroxysmal nocturnal hemoglobinuria (PNH) is an acquired hemopoietic stem cell disorder characterized clinically by chronic hemolytic anemia with acute episodes, thrombosis and bone marrow failure. It is a rare condition which usually occurs in younger people. Immunophenotyping and flow cytometry play a key role in diagnosing $\mathrm{PNH}$. Treatment is mainly supportive. Because it is so rare, delay in diagnosis is not uncommon in patients with $\mathrm{PNH}$, and this has a considerable impact on patient management and prognosis. We present this rare cause of hemolytic anemia, which should be considered in any patient, of any age, who has signs of chronic hemolysis.

\section{CASE REPORT}

A 29 year old gentleman who was working as a manual laborer in Saudi Arabia presented to the Medical Referral Clinic of Patan Hospital with the complaints of easy fatigability, exertional breathlessness and increasing pallor of one year duration. The patient had a few episodes of gum and nose bleed- ing prior to the presentation. However there was no easy bruisability, hematuria, hematemesis, or melena.

Before coming to our hospital, for the same complaints he visited a hospital in Saudi Arabia where he was diagnosed to have megaloblastic anemia. There he was treated with several injections of Neurobion and folic acid tablets. Though some symptomatic improvement occurred, his condition remained essentially the same and his hemoglobin never rose above $8 \mathrm{gm} / \mathrm{dl}$.

On reviewing the patient's history, there was no abdominal pain, vomiting or loose stools. He gave no history of drug intake apart from hematinics. There were no symptoms such as tingling and burning sensation or weakness of the extremities. There was no history of jaundice or alteration of urine color. He had no symptoms suggesting frequent infections, such as fever, cough, sore throat, skin eruptions, or burning urination.

On examination the patient appeared to be in relatively good health. His vital signs were within normal limits. Systemic examination was remarkable for moderate pallor and minimal

\footnotetext{
Address for correspondence :

Dr. Buddhi P. Paudyal

Patan Hospital, Lalitpur, Nepal

Email: buddhipaudyal@yahoo.com
}

Received Date : $13^{\text {th }}$ Jan., 2005

Accepted Date : $4^{\text {th }}$ April, 2005 
icterus. There were no significant lymph nodes, skin rashes, easy bruisability or bony tenderness. His central nervous system, cardiovascular system, and respiratory system were normal. Liver and spleen were not palpable and no masses were felt in the abdomen.

With these findings he was admitted for further work up. Investigations revealed hematocrit 24\%, WBC 2700/ml (Polys $60 \%$, Lymph 38\%, Mono 2\%), platelets 78,000/ml, reticulocytes 4.9\%, MCV $101 \mathrm{fl}$, and peripheral smear showing hypochromia and anisocytosis. Further investigations showed total bilirubin 2.1mg/dl (with direct 0.9), AST 65 unit/ 1, ALT 25 unit/l, alkaline phosphatase 63 unit/l and LDH 3105 unit/l. With these features of pancytopenia and hemolysis, bone marrow aspiration was performed, which revealed erythroid hyperplasia.

Investigations were done to find the underlying causes of hemolysis. The hemoglobin electrophoresis and G6PD level were normal. The direct Coomb's test, antinuclear antibody and VDRL were all negative. Ultrasound scan of the abdomen did not reveal any abnormal findings. The patient was referred to a medical in Vellore, India for further work up. Additional investigations there revealed positive urinary hemosiderin, and negative Ham's and sucrose lysis test. However immunophenotyping study showed diminished level of CD 59 in neutrophils (70\% of total) and erythrocytes (60\% of total). These latter findings confirmed the diagnosis of paroxysmal nocturnal hemoglobinuria in this case.

\section{DISCUSSION}

Paroxysmal nocturnal hemoglobulinuria ( $\mathrm{PNH}$ ) is a hematopoeitic stem cell disorder characterized by the formation of defective erythrocytes, granulocytes, and platelets. It is an acquired clonal hematological disorder, caused by a somatic mutation in the X-linked PIG-A gene which is required for the formation of phosphotidylinositol anchored proteins like CD55, CD59 and many others on erythrocyte surfaces. ${ }^{1}$ This disease was first described by Strubing in 1882 in a patient who presented with hemoglobinuria after a period of sleep. Later Marchiafava and Micheli worked out this disease in greater detail and for that reason this disease is also referred to as Marchiafava-Micheli Syndrome. ${ }^{2}$

PNH is often under-and delayed-diagnosed: It is rare, the history of hemoglobulinuria is often not elicited, and diagnostic tests are complicated and not widely available. ${ }^{3}$

The defect on the red cell surface i. e. a deficiency of CD 55, CD 59, and other proteins predisposes them to increased sensitivity to complement-mediated lysis resulting in intravascular hemolysis. The presence and extent of resultant hemoglobulinuria (the cola-colored urine) varies from person to person and may not be noted by the patient as it is quite irregular and occult. Granulocytes and platelets, like RBCs, show increased sensitivity to complement-mediated lysis. Activation of platelets by complement leads to platelet aggregation and thrombosis, usually in deep veins. Common sites of thrombosis include hepatic veins (Budd-Chairi syndrome), portal vein (portal hypertension), and cerebral veins (cerebral infarction). The proliferation of the PNH clone in the bone marrow decreases hematopoeitic stem cells, resulting in bone marrow failure and pancytopenia.

Our patient presented with progressive anemia unresponsive to hematinics, persistent pancytopenia and jaundice-which are all characteristic features of PNH. However he did not volunteer the history of passage of cola-colored urine. Since hemoglobinuria usually occurs at night and is often irregular, he might not have noticed it. Similarly our patient had no thrombotic episodes at the time of diagnosis. The serum level of soluble u-PAR (su-PAR) may be helpful to monitor the thrombotic events in $\mathrm{PNH}$ patients. ${ }^{4}$

The differential diagnoses of PNH includes the various causes of hemolytic anemia such as autoimmune, hemoglobinopathies, defects in RBC metabolism, and extra-RBC processes like hypersplenism. The presence of pancytopenia, hemosiderinuria, and features of thrombophilia helps to differentiate PNH from these other causes of hemolytic anemia. DIC could also present with all of these features, but it is commonly a more acute illness. The diagnosis of PNH is suspected when there is laboratory evidence of intravascular hemolysis, pancytopenia, and various degrees of erythroid hyperplasia, though marrow aplasia can also occur. The diagnosis is confirmed by flow cytometric demonstration of decreased CD 55 or CD 59 in erythrocytes and granulocytes. The Ham test, a commonly done diagnostic test in many laboratories, is difficult, time-consuming, and less reliable - as was shown by our patient's negative test.

The clinical course of PNH is quite variable. It usually runs a chronic course, and may evolve into aplastic anemia, myelodysplastic syndrome or even acute leukemia. Very rarely the abnormal clone may completely disappear and the patient be cured of the disease.

Currently available options for treatment of PNH include blood transfusions, iron therapy for urinary iron loss, and steroids to reduce haemolysis and thrombotic episodes. Anticoagulants are advocated for recurrent thromboses as well as prophylactically, but there is no clear evidence of benefit. Bone marrow transplantation can be considered curative, but the decision to recommend this treatment must take into account factors related 
both to PNH and to co-morbid conditions. Newer developments in treatment modalities include gene therapy (transducing stem cells with a functional PIG-A gene), auto transplantation (using stem cells selected for the expression of glycosyl phosphatidylinositol-anchored proteins), ${ }^{5}$ and specific inhibitors of complement. These are all challenges for the future.

\section{REFERENCES}

1. Shichishima $T$ and Noji H. A new aspect of the molecular pathogenesis of paroxysmal nocturnal hemoglobinuria Hematology. 2002 Aug; 7(4): 211-27.
2. Beutler E. Paroxysmal nocturnal hemoglobinuria. In: Williams Hematology.

3. Nathalong $\mathrm{O}$, Chuansumrit A, Prayoonwiwat W. Rapid screening of PNH red cell populations using the gel test. Southeast Asian J Trop Med Public Health. 2003 Dec; 34(4): 887-90.

4. Dong XY, Xu CG, Sun GR, Zhang T, Peng J. Expression of three kinds of GPI-anchor proteins in paroxysmal nocturnal hemoglobinuria, aplastic anemia and myelodysplastic syndromes patients and their clinical implications. Zhonghua Xue Ye Xue Za Zhi. 2004 Apr; 25(4): 198-201.

5. Meyers G, Parker CJ. Management issues in paroxysmal nocturnal hemoglobinuria. Int J Hematol. 2003 Feb; 77(2): 125-32. 